\title{
Improved techniques for monitoring the HF spectrum
}

\author{
James E. Giesbrecht ${ }^{a, b, c}$, Russell Clarke ${ }^{c}$, and Derek Abbott ${ }^{a, b}$ \\ ${ }^{a}$ Centre for Biomedical Engineering, The University of Adelaide, SA 5005, Australia \\ ${ }^{b}$ Dept. of Electrical \& Electronic Engineering, The University of Adelaide, SA 5005, Australia \\ ${ }^{c}$ Ebor Computing, 147 Henley Beach Rd., Mile End, SA 5031, Australia
}

\begin{abstract}
A critical review of contemporary papers on modulation recognition, signal separation, and Single Station Location (SSL) is described in the context of High-Frequency (HF) radio-communications. High-frequency communications is undergoing resurgence despite advances in long-range satellite communication systems. Defense agencies are using the HF spectrum for backup communications as well as for spectrum surveillance applications. Spectrum management organizations are monitoring the HF spectrum to control and enforce licensing. This type of activity usually requires a system that is able to determine the location of a source of transmissions, separate valid signals from interferers and noise, and characterize signals-of-interest (SOI). The immediate aim is to show that commercial-of-the-shelf (COTS) equipment can be used to locate HF transmission sources, enhance SOIs and reject interference, and recognize signal types. The described work on single-station-location (SSL), signal separation, and modulation recognition is contributing to these goals. This paper describes the overall objectives and some of the disadvantages and benefits of various schemes for single-station-location (SSL), signal separation, and modulation recognition. It also proposes new approaches that may relieve shortcomings of existing methods - including selection of benchmarks or modulations for various transmission scenarios and propagation modes, and use of multiple digital receivers or compression techniques to improve modulation recognition, signal separation, and location of HF emitters.
\end{abstract}

Keywords: SSL, Spectrum Monitoring, HF, Modulation Recognition, Signal Separation

\section{INTRODUCTION}

This paper critically reviews contemporary papers on modulation recognition, signal separation, and Single Station Location (SSL) in the context of HF radio-communications. The HF radio band (nominally $2 \mathrm{MHz}$ to $30 \mathrm{MHz}$ ) can be used for long-distance wireless communications because the ionosphere and its various layers refract transmissions in this band. Such refraction enables signals to propagate beyond the horizon to distant receivers unable to be reached by higher frequency (VHF and above) signals. This fact makes the HF band attractive for private and commercial interests as well as for defense forces spread across the globe. For example, spectrum management agencies monitor the HF band for unlicensed operators and military agencies use the HF band for communications.

In the past, $\mathrm{HF}$ communication systems were analog. This meant that signals received by an antenna were down-converted to baseband using filters, oscillators, and many discrete components. Receivers were generally constructed in a super-heterodyne configuration. ${ }^{1}$ The baseband signals were then passed through demodulators to extract the information content. Often special demodulators had to be switched in to demodulate signals with different modulation schemes. Then of course in military scenarios, there were jamming signals to contend with and the need to determine the location of the source of a transmission. Depending on the complexity of the receive system, one or perhaps two interfering signals could be rejected while a beam was formed in the direction of a signal-of-interest (SOI). This would have been done with analog computation techniques, but with the advent of the digital computer many of these tasks became less cumbersome.

Send correspondence to:

The University of Adelaide - E-mail: james@eleceng.adelaide.edu.au; Phone: +61 (8) 83036296

Ebor Computing - E-mail: james.giesbrecht@ebor.com; Phone: +61 (8) 8351 7122; Fax: +61 (8) 83517079 
Today, there are many different modulation techniques. Many of these are of the family of space-time layered signals. Some techniques include direct-sequence spread spectrum (DSSS), frequency-hopped spread spectrum (FHSS), time-domain multiplexing (TDM), frequency-domain multiplexing (FDM), and parallel transmission of data through multiple antennas and/or frequencies. Traditional HF monitoring, processing, and analysis cannot easily handle these signals.

Monitoring and detection of such signals using traditional methods would require numerous HF receivers. Monitoring and detection would also require some prior knowledge of the signals so as to choose the correct receiver. This is a difficult task. Furthermore, recognition of a particular SOI amongst other signals is extremely complicated with analog techniques. Early computers made these processes easier, but only recently has enough processing power been available in one package to perform all the tasks above.

Today software radio aims to replicate hardware functions in software running on a generic platform. In so doing many of the problems associated with hardware implementations are avoided. In addition, the receiver and transmitter chains can easily be changed to accommodate various modulation schemes. The idea is to digitize the incoming radio-frequency ( $\mathrm{RF}$ ) signal directly and then to perform down-conversion and demodulation in digital hardware. High resolution Analog-to-Digital Converters (ADCs) and Digital Down-Converters (DDCs) are now available for operation at sampling rates in the $100 \mathrm{MHz}$ range. These sampling rates allow for direct digitization of RF signals up to around $50 \mathrm{MHz}$.

This work consists of the research and development of a digital HF receiver to demonstrate capability to perform a number of tasks, some of which include recognition of signal modulation, spatial and temporal separation of co-channel signals, single station location (SSL) through direction finding algorithms, rejection of sources of interference, and enhancing signals-of-interest (SOIs). The research focuses on the first three objectives. Another objective is to demonstrate that commercial-of-the-shelf (COTS) equipment, such as data acquisition cards and inexpensive computers, can be configured to satisfy the above objectives and to provide superior performance to current HF monitoring equipment at a significantly lower cost.

\section{METHODS}

Though there are many papers on the topics of modulation recognition, signal separation, and SSL most provide only simulation results or do not adequately consider operation of the techniques in the HF band. What follows is a brief overview of recent research in these areas.

\subsection{Modulation Recognition}

The process of determining the modulation type of a signal with no foreknowledge of the signal modulation characteristics is known as modulation recognition. This field has been a topic of research since the mid-1980s. During those early days, modulation recognition was accomplished through multiple hardware demodulators one for each modulation type of interest. With the advent of software radio, these multiple demodulators are being combined in software. With either method, the purpose of modulation recognition is to determine the type of modulation so that the correct demodulator can be chosen to demodulate the signal.

Fundamental processes of modulation recognition are feature extraction and classification (see Figure 1). Feature extraction determines unique characteristics of the signal so that a classifier can establish the modulation type. Common features include instantaneous amplitude, variance of phase, spectral symmetry, transmission models, and higher order statistics. Classification associates features with modulation types. These associations are normally made through threshold detection logic, artificial neural networks (ANNs), or pattern recognition algorithms.

Hero $^{2}$ and Nolan et $a l^{3}$ described statistical methods for feature extraction and used threshold detection logic for feature classification. Their methods were able to correctly recognize various types of phase-shift-keying (PSK) and frequency-shift-keying (FSK). However, their assumptions of additive white Gaussian noise (AWGN), ideal bandpass filtering, Rayleigh fading, Doppler effects, or uniformly distributed phase noise are not generally valid for HF communications. Nor have they included in their papers any field-testing of their methods. Ketterer ${ }^{4}$ et $a l$ also applied statistical methods and threshold detection to tackle the modulation recognition problem. But 


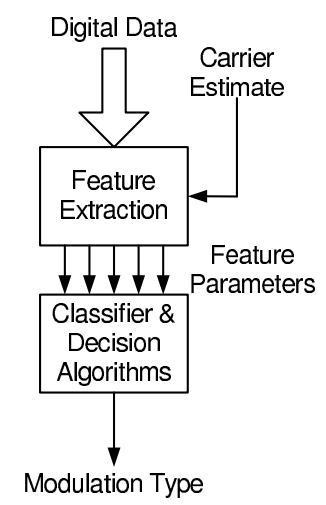

Figure 1. A typical modulation recognition structure.

in this case, they demonstrated the robustness of their algorithm by applying it to a real-world short-wave radio signal having symbols identical to their synthetic data.

Others have taken a hybrid approach to modulation recognition. Wong and Nandi ${ }^{5}$ described a feature extraction and classification algorithm for discriminating PSK, FSK, V.29, V.32, and QAM. Feature extraction was obtained through statistical methods, while classification was based on ANNs. Without noise and with suitable training, their method was capable of recognizing the modulation types with no error. With simulated noise, their algorithm was able to recognize the modulation types with about $10 \%$ error. The authors, however, do not mention the noise model for their simulations and do not consider the effects of real-noise, multi-path, or Doppler shifts on the performance of the system. All of these strongly influence HF communications and so it is doubtful that the performance figures they quote are achievable for practical HF transmissions.

Waller and Brushe ${ }^{6}$ addressed the problem of modulation recognition in a completely different way. Instead of pattern recognition, parameter estimation, or neural networks, the authors chose the best model of the transmission system based on observations of the noisy received signal. They focused on frequency modulation (FM) and phase modulation (PM) and used a cross-correlation approach that gave rise to characteristic peaks for FM or PM. However, their identification of correlation peaks relies on appropriate threshold detection and in this sense, their method is no different from many other researchers. ${ }^{2,3,7}$

The modulation recognition methods presented above do not adequately rationalize the choice of thresholds and feature functions. Moreover, they do not assume appropriate noise and channel models ${ }^{8,9}$ for application of their methods to the HF band, and they do not apply their methods to real signals (cf. Ketterer ${ }^{4}$ ).

\subsection{Signal Separation}

Signal separation is commonly performed in the time, frequency, and spatial domains. Temporal/spectral separation uses time/frequency characteristics of the observed signal to break it into its constituent parts. Spatial separation relies on differences in physical location of signal sources to decompose a received signal into its components.

Figure 2 illustrates the concept of non-spatial signal separation. Numerous sources transmit their signals over the radio channel, which is affected by fading, noise, Doppler shifts as well as multi-mode propagation and multi-path effects. The job of the receiver is to decompose the received signal into its parts.

Jang and Lee ${ }^{10}$ demonstrated a method to separate multiple audio sources from one observed audio signal based on linear combinations of sound sources and basis functions. Their method maximized the closeness of linear combinations of the audio sources to the real observed signal. They found that a mixture of jazz music and male speech was more easily separated than other combinations of rock music, jazz music, and male and female speech. However, their method requires a set of statistically independent basis functions, which may be particularly difficult to choose for radio-communications because of the many different digital and analog signal types. 


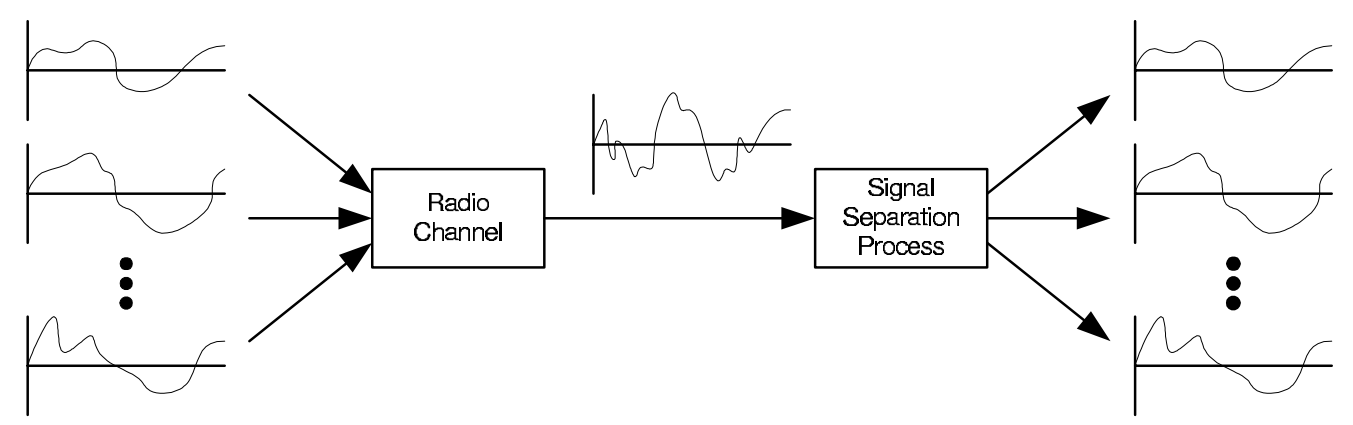

Figure 2. The basic signal separation technique.

Stanacevic et $a l^{11}$ proposed a method to simplify the separation of mixed signals arriving at a miniature microphone array. The technique reduced the received signal to a set of spatial and temporal differentials which were used to estimate the direction-of-arrival of the source signals and to thereby separate them from the combined received signal. They claimed a $12 \mathrm{~dB}$ to $20 \mathrm{~dB}$ reduction in cross-talk between individual sound sources received by the array. The principles of their algorithm could be applied to HF radio-communications, but their assumptions of far-field analysis (in this case tens of centimeters), statistical independence, and Gaussian noise do not apply. Propagation of HF signals is affected by non-Gaussian noise, variations in the ionosphere, and multi-path effects. Far-field assumptions for HF signals require distances of tens of kilometers.

Chan, Rayner, and Godsill ${ }^{12}$ considered $n$ independent source signals detected by $n$ sensors. The problem they faced was to find $n^{2}$ separating functions to extract the original $n$ source signals from the composite received signals. They developed a cost function and minimized it with respect to the separating functions. Their algorithm was able to separate an estimate of each source signal but could not extract the true source signals because the extracted signals were an arbitrary function of the source signals. This method too, could be adapted for the HF environment but it would require removal of their assumption of signal independence.

Ham and Faour ${ }^{13}$ conducted analyses of infrasound signals less than $2 \mathrm{~Hz}$ from volcanic eruptions, gravitational waves, and mountain associated waves. They used Independent Component Analysis (ICA) to separate unknown source signals from the linear mixtures received by multiple sensors and processed by a neural network. Based on the assumption of zero-mean wide-sense stationary source signals, they estimated the corresponding ICA basis vectors. Individual signals could then be separated from a random but linear combination of the signals. But, the assumption of zero-mean wide-sense stationary signals is not always valid for radio-communications. In addition, their work at frequencies less than $2 \mathrm{~Hz}$ allows the neural network to be trained very quickly whereas operation in the HF band may dictate long training periods.

Yen and Zhao, ${ }^{14}$ Park et al, ${ }^{15}$ and Erten and Salam ${ }^{16}$ have focused on signal separation for speech recognition, but their methods could find use in HF applications. Yen and Zhao proposed a method for adaptive de-correlation filtering (ADF). The ADF method attempts to de-correlate speech signals by comparing the variances and covariances of estimates of the source signals with defined thresholds in the time and frequency domains. Erten and Salam proposed a state-space method for blind signal separation of voice that utilized a dynamic mixing matrix instead of the common static matrix. This had the advantage of addressing signal propagation delays, nonlinear mixing functions, and unknown numbers of source signals which the static mixing matrices could not handle.

Much signal separation research suffers inadequate or incorrect assumptions and lack of field-testing, if the methods are to be applied to HF communications. HF radio requires that assumptions of signal independence, Gaussian noise, and uncorrelated signals be reviewed. Characteristics of the ionosphere and re-radiating structures can cause signals in the HF band to appear statistically dependent and correlated, and to propagate by multiple modes. HF signals are also subject to significant fading, Doppler, and multi-path effects. 


\subsection{Single Station Location (SSL)}

Single Site Location (SSL), which is also called Single Station Location, is a method that estimates the bearing and range of a transmission source given measurements from a single receiver site. A general SSL system consists of multiple antennas, an ionosonde, and processing system. The arrangement of the antennas is such that differences in signal phase between antennas, and ionospheric information, translate to bearing and elevation information.

Treharne, ${ }^{17}$ Gething, ${ }^{18}$ and Groller ${ }^{19}$ provide brief commentaries on range and bearing determination. The most common non-SSL method is to fix the position of an emitter based on estimates of bearing from three or more receive sites. Such horizontal triangulation methods, however, require sophisticated coordination between receive sites as well as information about the ionosphere along each of the ray paths. SSL uses its antenna array to estimate bearing and elevation while it performs triangulation in the vertical plane (see Figure 3) to estimate range. Given a measurement of elevation angle $\beta$, and reflection height $h$, the range $R$ is

$$
R=2 d=\frac{2 h}{\tan \beta} .
$$

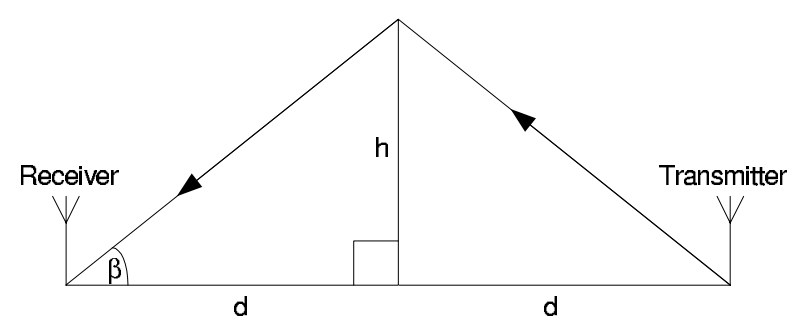

Figure 3. A simple model for range calculation in SSL.

In general, range and bearing estimation suffers from the uncertainties of the ionosphere. These uncertainties include ionospheric tilts, Traveling Ionospheric disturbances (TIDs), variations in refraction regions (e.g. D, $\mathrm{E}$, and $\mathrm{F}$ layers), multiple simultaneous propagation modes, and difficulties in identifying modes and reflection height. Consequently, accurate determinations of range and bearing require knowledge of the ionosphere along the ray path. This knowledge would ideally come from multiple vertical-incidence (vi-) ionosondes or an obliqueincidence (oi-) ionosonde. But, usually only one vi-ionosonde is present at an SSL receive site to determine ionosphere height and tilt. Predictions of the characteristics of the ionosphere along the rest of the ray path come from appropriate ionospheric models.

Even so, McNamara ${ }^{20}$ showed that range determination by vertical triangulation could be quite accurate (errors of the order of 10\%) provided an appropriate ionospheric model was chosen. McNamara found that errors in bearing and range measurements were almost exclusively limited by ionospheric effects, and that a simple quasi-parabolic ionospheric model would suffice in lieu of real-time monitoring of ionospheric conditions.

Most SSL systems can determine transmitter location up to ranges of approximately $2500 \mathrm{~km}^{17,19,20}$ but perform poorly beyond this distance because of their inability to accurately resolve signal modes (propagation paths). Bradley, Damboldt, and Suessmann ${ }^{21}$ discussed available modelling options for determining HF propagation modes. They listed numerous shortcomings of existing methods such as: inaccurate ionospheric models for large regions of the world, improperly addressed spatial and temporal ionospheric variations at high latitudes, poorly modelled ionospheric irregularities, too few ionospheric soundings, and uncertainties in communication equipment. They suggested that schemes must be found that 1) improve the accuracy of current methods, and 2) that are simpler than current methods.

Goodwin et $a l^{22}$ started along the road to better techniques when they indicated that single-mode waves provided nearly linear wavefronts for good bearing determination. Multi-mode signals created a non-linear wavefront at an antenna array and tended to fade. They suggested that a low-bandwidth bi-phase modulation method could reduce the negative effect of multi-mode signals. 
Numerous other papers ${ }^{23-25}$ tout the benefits of particular modulation schemes to combat multi-path and fading in the HF band, but all focus on improving Bit-Error-Rate (BER) performance and all assume that a transmitter and receiver are cooperating in the communication. An uncooperative transmitter would be more difficult to locate.

Baker $e t a l^{26}$ proposed a technique for the decomposition of modes based on phase and amplitude information extracted from the time varying transfer function of an HF sky-wave. They swept the transmit frequency of an oblique sounding link over a narrow bandwidth and recorded the received baseband signals. By applying the Fourier Transform to odd and even functions of the received signals, they were able to extract the phase component of the received signals. Baker et al concluded that individual signal modes could be separated and intermodal interference recognized, but additional signal processing techniques based on null-steering and adaptive polarization were necessary to reduce phase distortion and thereby improve modal separation in some situations.

Performance of SSL systems is highly dependent on the conditions of the ionosphere. The main areas of concern are the separation of propagation modes, and adoption of accurate models of the ionosphere along the ray path. A number of researchers suggest that various modulation schemes and signal separation techniques can be used to improve SSL performance. These techniques may also improve the discernment of propagation modes.

\section{IDEAS TO IMPROVE METHODS}

Except for the SSL area, the above survey identifies that there are very few papers on modulation recognition or signal separation in the context of HF communications. For modulation recognition, most research is conducted with synthetic data and frequencies much higher than the HF band. Research in signal separation appears to mainly concentrate on speech recognition applications. Within the SSL field, the main problems appear to be the unpredictability of the ionosphere and the inability to adequately identify propagation modes. This section describes intended research in areas.

\subsection{Modulation Recognition}

Current research on modulation recognition concentrates on trial-and-error and statistical methods, threshold detection logic, pattern recognition techniques, or artificial neural networks. A number of these have been described above. All perform two basic functions: feature extraction and signal classification; and all suffer a common problem.

The problem is that of practical validity. Many researchers claim success of their methods based on assumptions of additive-white-Gaussian-noise, propagation characteristics, simulated data, and somewhat arbitrary thresholds. These assumptions may be justified in theory but are not generally valid in practice.

Validation raises many questions. Are the chosen correlation thresholds valid for HF communications? What is the real noise distribution for the HF band? Are the assumed propagation characteristics valid in all situations? What is the best way to determine the modulation type? Are brute-force methods better than statistical methods? Is there a benchmark signal or benchmark noise distribution that could be used to verify modulation recognition methods for the HF band? If a recognition technique fails in the field, why did it fail, and how can it be improved to operate successfully? Which recognition method is the most robust and able to handle real data?

A medium-term goal is to answer these questions and to apply the answers to known and new modulation recognition algorithms. A part of present work is focused on selecting a set of benchmarks (drawn from real HF signals), characterizing the benchmarks, and then using a table of characteristics to identify an arbitrary HF signal. The ultimate goal is to provide figures for recognition rate for a particular HF signal against benchmark and transmission environment. One such benchmark is that of signal entropy. Benedetto et al ${ }^{27}$ discuss an interesting method that, with suitable modification, may be a candidate for recognition of HF modulation types.

Benedetto, Caglioti, and Loreto ${ }^{27}$ presented a method for extracting information from generic sequences. In their paper, the researchers described a way to measure the relative entropy between two information streams 
$\boldsymbol{A}$ and $\boldsymbol{B}$. They compressed a long sequence $A$ from $\boldsymbol{A}$ and subtracted the compressed length from the length of the compressed sequence $A+b$, where $A+b$ was the concatenation of $A$ and a small sequence $b$ from $\boldsymbol{B}$. This was defined as the entropy of $\boldsymbol{A}$ (designated by $\Delta_{A b}$ ). In a similar manner they computed the entropy of $\boldsymbol{B}$ as $\Delta_{B b}$. They then defined the relative entropy between the two information sequences as

$$
S_{A B}=\frac{\Delta_{A b}-\Delta_{B b}}{|b|}
$$

and applied the algorithm to language recognition, authorship attribution, and classification of sequences. In each application the measure of relative entropy was successful in identifying a language, author, or language classification. For example, the method was able to successfully distinguish between Dutch, Danish, English, French, Finnish, German, Italian, Portuguese, Spanish, and Swedish. It was also able to correctly identify the author of a text $93.3 \%$ of the time. And for language classification, the method was able to correctly classify the romance, celtic, germanic, slavic, and baltic languages.

This method is general enough to apply to HF modulation schemes and is now being tested on various HF signals* at varying signal-to-noise ratios (SNRs) and in different transmission environments. The entropies of synthetic versions of the HF signals are being compared to the entropies of real benchmark HF signals to derive a table of entropies that could be used to identify an arbitrary HF signal. From this table a robust modulation recognition algorithm is being developed that is tailored for $\mathrm{HF}$ communications.

\subsection{Signal Separation}

Many researchers attack the signal separation issue with independent component analysis (ICA) techniques. ICA is a statistical method for analyzing hidden facets of random variables, measurements, or signals. In ICA, data variables are estimated based on a large sample space of observations, and linear or non-linear combinations of those observations. The mixing function that combines the observations is also unknown. With the assumption that the data variables are Gaussian and mutually independent, ICA can extract independent components of the input signals and thereby separate signals.

Another common thread to signal separation is the assumption of independent and uncorrelated inputs. For the most part, different signal sources in the HF band are independent. However, if propagation of a signal occurs by multiple layers of the ionosphere, the multi-path signals at the reception point will be correlated. They may or may not be independent. The idea then, is to see what amount of correlation and independence of HF signals will cause the algorithms to fail or succeed. It appears that few researchers have considered this aspect of their algorithms.

In this part of the author's research, a nearby transmitter sends a direct HF ground-wave to the digital receiver mentioned in Section 4. It will also have a direct connection (e.g. by terrestrial link) to a distant station and be capable of cleanly re-transmitting the broadcast of the distant transmitter or mixing the clean re-transmission with an interfering signal through a mixing function. The re-transmission serves as a reference signal because it is an undistorted ground-wave that can be assumed to be merely attenuated and delayed. The signal from the distant transmitter is received by HF sky-waves. These sky-waves arrive at the receiver by multiple propagation modes, different paths (multi-path), variable attenuation and delays, fading, frequency shifts and spreading, time dispersion, and delay distortion created by the ionosphere and other structures.

A correlation coefficient then measures the similarity between each mode of the sky-wave and reference signal. To do this, the nearby transmitter adjusts its transmission in such a way as to vary the correlation coefficient. The objective is to determine the success rate of a number of the signal separation algorithms against the correlation coefficient.

In a like manner, an independence coefficient is of use. Inherent in a measure of statistical independence are the distributions of the signals. Observations of the reference, interferer, sky-wave, and mixed signal provides their distributions. The next step is to compare the sky-wave and its distribution with the reference to determine the effect that the ionosphere has on independence. Then, by adjusting the weighting of each input of a mixing

*AM Voice, STANAG 4285, ANDVT 39-Tone, MIL-STD-188-110A, and MIL-STD-188-141A. 
function the level of independence between the sky-wave and mixed signal will change. A number of these changes will yield a graph of success rate of separation algorithms versus independence coefficient.

The author also intends to investigate the efficiency of the separation algorithms. In this case, the nearby transmitter will transmit an interferer while the digital receiver acquires the sky-wave broadcast and the interferer. The acquired data will be processed by the separation algorithms to determine their success rate of separation versus Signal-to-Interference Ratio (SIR).

\subsection{Single Station Location (SSL)}

The performance of SSL systems is limited by the characteristics of the ionosphere. Ionospheric tilt, traveling ionospheric disturbances, uncertainty of ionosphere height, and other unknown conditions along the transmission path affect the accuracy of determining location of a distant transmitter. And, the inability of SSL systems to adequately detect arriving signal modes further complicates the calculation of transmitter position.

Common methods to address these issues include ionospheric models that assume stationary characteristics over a large area of the earth, estimates of variations of the ionosphere with altitude, modeling of ionospheric irregularities, and selected ionospheric soundings over portions of the earth. These techniques, though used for many years, are cumbersome and often inaccurate. There needs to be an easy and efficient procedure to determine the properties of the ionosphere along a transmission path.

One idea is to compare vertical-incidence (vi-) and oblique-incidence (oi-) ionograms to create a synthetic ionogram that can be related to the results of a real-time vi-ionosonde (see example in Figure 4) at an SSL receiver. The plan to investigate this idea requires a distant HF transmitter, an SSL receiver (see Section 4), and vi- and oi- ionosondes. An oi-ionosonde transmitter, located at the distant HF broadcast transmitter, transmits a signal to an oi-ionosonde receiver situated near the SSL receiver. The resulting ionogram (see example in Figure 4) will show the properties of the ionosphere along the path between the HF transmitter and SSL receiver. The vi-ionosonde determines the properties of the ionosphere above the SSL receiver. If the distant HF transmitter then sends a signal to the SSL receiver, the properties of the received signal and the two ionograms can aid the creation of a synthetic oi-ionogram for various ionospheric conditions. These synthetic ionograms will enable more accurate inference of the condition of the ionosphere between two points given a local vi-ionogram.
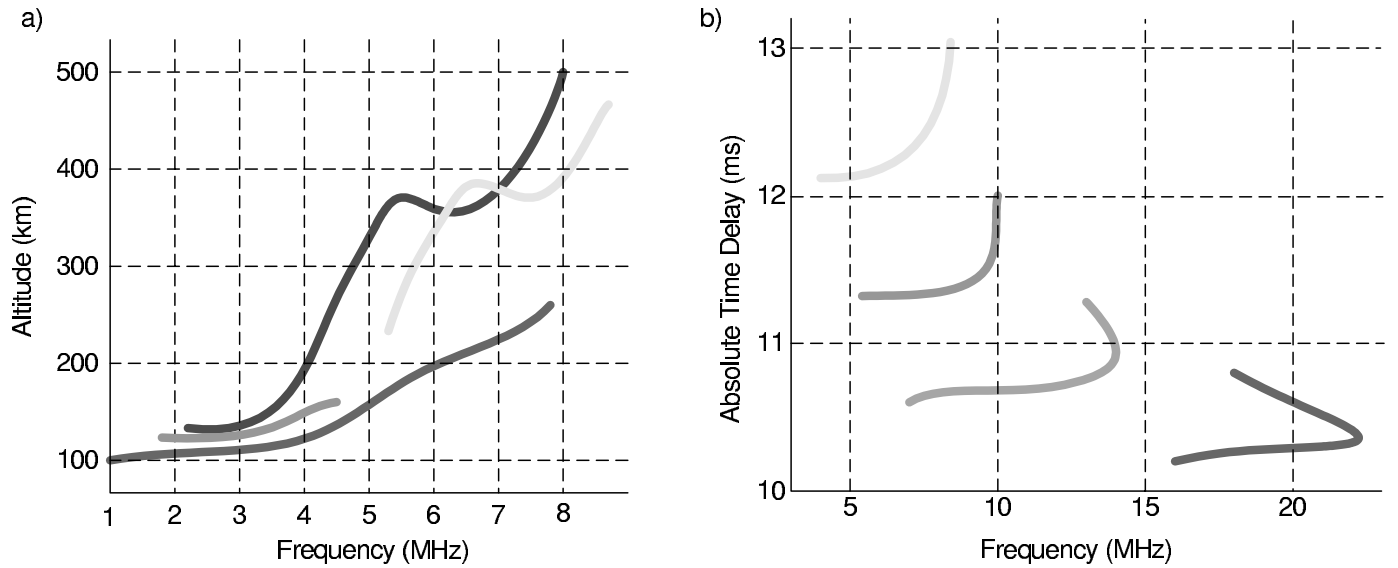

Figure 4. A typical a) vertical incidence ionogram and b) oblique incidence ionogram.

Another theory is that signal separation techniques improve the ability of SSL systems to recognize and resolve signal modes. A local vi-ionogram and a synthetic oi-ionogram along the propagation path may help to predict the signal modes arriving at the SSL receiver and the height of reflection. Signal separation techniques could then extract the dominant mode for an accurate range determination, which depends on the dominant mode and reflection height. For instance, the methods of Yen $^{14}$ and Erten ${ }^{16}$ may be adapted to separate the expected modes based on the predictions of the synthetic ionogram. Recombining the estimated modes and 


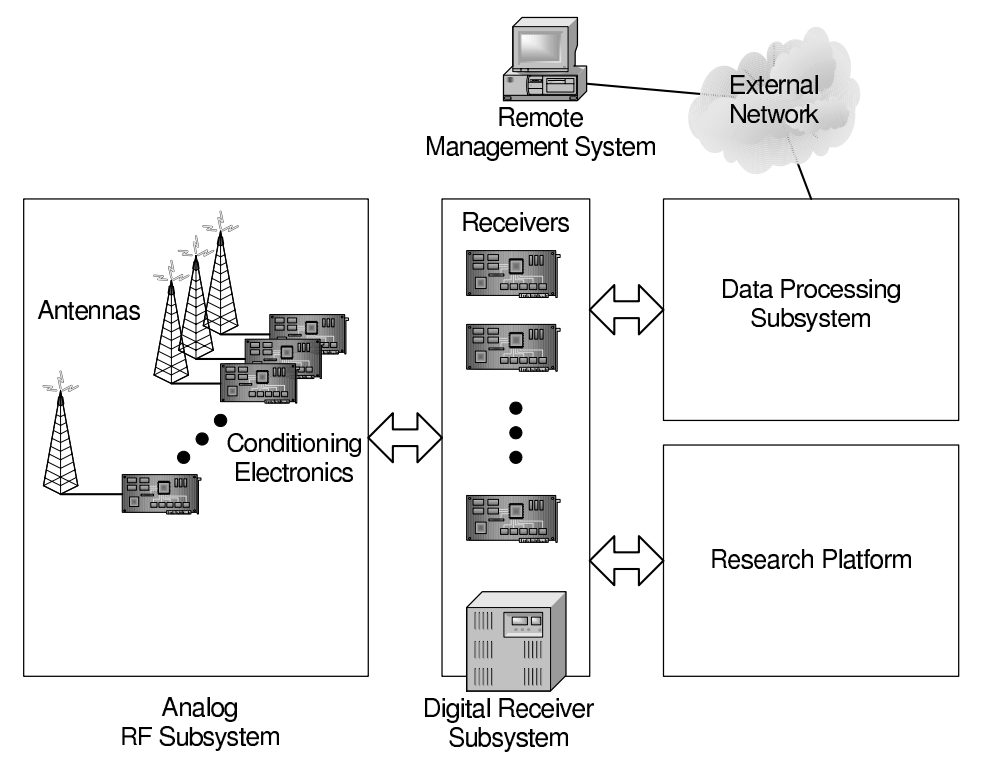

Figure 5. Architecture of the HF monitoring system.

correlating with the actual incoming signal will show how well the separation algorithms are able to decompose the original signal into its constituent modes. A subsequent comparison between bearing and range calculations on the estimated modes and the original signal and the true bearing and range of the transmitter will demonstrate the performance gain or loss of the method. If signal separation techniques do improve SSL performance, one would expect to see that the bearing and range calculation on the estimated signal modes is more accurate than the calculation on the original distorted signal.

Finally, modulation type and equalization may play a role in mitigating the effects of the ionosphere on HF transmissions and therefore the accuracy of range and bearing calculations. Using the oi-ionosonde and HF transmitter, mentioned above, a study of modulation and equalization techniques will demonstrate the most appropriate modulation and equalization techniques for a given ionospheric scenario. Such a study will consist of characterizing the ionosphere and its affect on a benchmark HF transmission having different modulations. For example, during one ionospheric scenario it may be that the location of HF transmissions utilizing FSK are more easily discerned than similar transmissions using amplitude modulation. Also, it may be that one equalization technique is more useful for SSL than another. Furthermore, it may be that resolving propagation modes of signals having a particular modulation scheme is easier than for signals of a different modulation scheme.

\section{RESEARCH PLATFORM}

An HF Monitoring System and research platform is currently under development at Ebor Computing. This monitoring system consists of an array of antennas, followed by signal conditioning electronics, multiple digital receivers, and a data processing sub-system (see Figure 5).

The Analog RF Subsystem consists of antennas, amplifiers, attenuators, and filters. Outputs of this subsystem are fed to a rack of digital receivers that directly sample RF signals up to $50 \mathrm{MHz}$ and down-convert the $\mathrm{HF}$ channels to baseband. The digitization of the RF signals is achieved by high-speed ADCs. Down-conversion is accomplished with DDCs. The Data Processing Subsystem collects the baseband information and processes it. Processing includes functions such as modulation recognition, signal separation, and SSL. A Remote Management System oversees the operation of the entire system. The research platform operates independently of the Data Processing Subsystem and Remote Management System. It is used to collect non-proprietary signals and to implement algorithms such as those previously mentioned. 


\section{SUMMARY AND FUTURE WORK}

The previous discussion identifies a number of problems with recent research in modulation recognition, signal separation, and SSL. For modulation recognition, the problems stem from inadequate assumptions about the nature of the noise of the transmission medium and the propagation characteristics, as well as a reliance on arbitrary thresholds and synthetic data. Current signal separation research appears to concentrate mainly on speech recognition/separation applications. Much of this research assumes statistically independent and uncorrelated signals as well as Gaussian noise. None of these assumptions are generally valid in HF communications. SSL research, though vast, has still to solve the problem of accurately separating dominant propagation modes from an incoming signal and adequately modeling the characteristics of the ionosphere along the propagation path.

The immediate work effort is the development of an HF Monitoring System and research platform (see Section 4). Some aspects of the effort relate to modulation recognition, signal separation, and SSL. Software for the generation of synthetic HF signals is also being created as a part of the modulation recognition research. Future work will use the research platform to collect real HF signals (groundwave and skywave) and to process them with some of the presented algorithms.

\section{ACKNOWLEDGMENTS}

Financial support for this work is provided by the The University of Adelaide and Ebor Computing.

\section{REFERENCES}

1. J. Smith, Modern Communication Circuits, McGraw-Hill Series in Electrical Engineering, McGraw-Hill Inc., New York, 1st ed., 1986. pp. 1-13.

2. A. O. Hero III and H. Hadinejad-Mahram, "Digital modulation classification using power moment matrices," in Proceedings of the IEEE 1998 International Conference on Acoustics, Speech, and Signal Processing, (Seattle), 1998.

3. K. E. Nolan, L. Doyle, P. Mackenzie, and D. O'Mahony, "Modulation scheme classification for 4G software radio wireless networks," in IASTED International Conference on Signal Processing, Pattern Recognition, and Applications (SPPRA 2002), (Crete, Greece), 2002.

4. H. Ketterer, F. Jondral, and A. H. Costa, "Classification of modulation models using time-frequency methods," in Proceedings of the IEEE 1999 International Conference on Acoustics, Speech, and Signal Processing, (Phoenix, Arizona), 1999.

5. M. L. D. Wong and A. K. Nandi, "Automatic digital modulation recognition using spectral and statistical features with multi-layer perceptrons," in Proceedings of the IEEE International Symposium on Signal Processing and its Applications, 2, pp. 390 - 393, (Kuala Lumpur, Malaysia), 2001.

6. J. R. Waller and G. D. Brushe, "A method for differentiation between frequency and phase modulated signals," in Proceedings of IEEE Information, Decision, and Control 1999, 1999.

7. H. C. Tan, K. Sakaguchi, J.-I. Takada, and K. Araki, "DOA based Signal Combining aided Automatic Modulation Recognition/Demodulation for Surveillance System," tech. rep., The Institute of Electronics, Information and Communications Engineers (IEICE), 2002.

8. L. Sevgi and A. M. Ponsford, "An HF Radar Based Integrated Maritime Surveillance System," tech. rep., Raytheon Systems Canada Ltd, 1999. http://www3.dogus.edu.tr/lsevgi/LS_PROF/SYMP/siu99.pdf.

9. M. Goris, "Categories of Radio-Frequency Interference," tech. rep., National Foundation for Radio Astronomy, Holland, February 1998.

10. G.-J. Jang and T.-W. Lee, "A probabilistic approach to single channel blind signal separation," in Proceedings of the 15th Conference on Neural Information Processing Systems: Natural and Synthetic 2002 (NIPS2002), Neural Information Processing Systems, (Vancouver, Canada), 2002.

11. M. Stanacevic, G. Cauwenberghs, and G. Zweig, "Gradient flow adaptive beamforming and signal separation in a miniature microphone array," in Proceedings of the IEEE International Conference on Acoustics, Speech, and Signal Processing (ICASSP'2002), (Orlando, Florida), 2002. 
12. D. C. B. Chan, P. J. W. Rayner, and S. J. Godsill, "Multi-channel signal separation," in Proceedings of the IEEE 1996 International Conference on Acoustics, Speech, and Signal Processing, 2, pp. 649 - 652, (Atlanta, Georgia), May 1996.

13. F. M. Ham and N. A. Faour, "Infrasound signal separation using independent component analysis," in 21st Seismic Research Symposium, 133, Los Alamos National Laboratory - LA-UR-99-4700, (Las Vegas, Nevada), 1999.

14. K.-C. Yen and Y. Zhao, "Robust automatic speech recognition using a multi-channel signal separation frontend," in Proceedings of the IEEE 4th International Conference on Spoken Language Processing, 3, pp. 1337 - 1340, 1996.

15. H.-M. Park, H.-Y. Jung, T.-W. Lee, and S.-Y. Lee, "Subband-based blind signal separation for noise speech recognition," Electronic Letters 35(23), pp. 2011-2022, 1999.

16. G. Erten and F. M. Salam, "Voice extraction by on-line signal separation and recovery," IEEE Transactions on Circuits and Systems - II: Analog and Digital Signal Processing 46(7), 1999.

17. R. F. Treharne, "Non-military applications of high frequency single station location system," Journal of Electrical and Electronics Engineering, Australia 1(1), pp. 87 - 92, 1981.

18. P. J. D. Gething, Radio Direction Finding and Super-resolution, IEE Electromagnetic Waves Series 33, Peter Peregrinus Ltd, 2nd ed., 1991.

19. R. Groller, "Single station location HF direction finding," Journal of Electronic Defense 13(6), pp. 58 - 63, 83, 1990.

20. L. F. McNamara, "Ionospheric modelling in support of single station location of long range transmitters," Journal of Atmospheric and Terrestrial Physics 50(9), pp. 781 - 795, 1988.

21. P. A. Bradley, T. Damboldt, and P. Suessmann, "Propagation models for HF radio service planning," in IEE Eighth International Conference on HF Radio Systems and Techniques, 474, pp. 175 - 179, (London), 2000.

22. G. L. Goodwin, Z. R. Jeffrey, and D. J. Hichens, "A high frequency radio location system," Journal of Electrical and Electronics Engineering, Australia 12(3), pp. 284 - 289, 1992.

23. W. N. Furman, "Robust low bit rate hf data modems," in IEE Seventh International Conference on HF Radio Systems and Techniques Conference, 441, pp. 149 - 153, (London, UK), 1997.

24. C. J. Demeure and P. A. Laurent, "A new modem for high quality sound broadcasting at short waves," in IEE Seventh International Conference on HF Radio Systems and Techniques Conference, 441, pp. 50 - 54, (London, UK), 1997.

25. A. P. Noble, J. A. Spicer, M. P. Midwinter, and S. G. Farquhar, "The viability of very high data rate modems for a specific HF channel type," in IEE Seventh International Conference on HF Radio Systems and Techniques Conference, 441, pp. 55 - 59, (London, UK), 1997.

26. P. W. Baker, R. H. Clarke, A. D. Massie, and D. Taylor, "Techniques for the measurement and decomposition of the time varying narrow bandwidth transfer function of an HF sky wave transmission," Radio Science 32(5), pp. 1813 - 1820, 1997.

27. D. Benedetto, E. Caglioti, and V. Loreto, "Language trees and zipping," Physical Review Letters 88(4), pp. $048702-1$ to $048702-4,2002$. 